\title{
Review
}

Journal of Innate

Immunity
J Innate Immun 2011;3:28-33

DOI: $\underline{10.1159 / 000321931}$
Received: August 29, 2010

Accepted after revision: October 12, 2010

Published online: November 6, 2010

\section{Role and Importance of Phenoloxidase in Insect Hemostasis}

\author{
Ioannis Eleftherianos Christina Revenis \\ Department of Biological Sciences, The George Washington University, Washington, D.C., USA
}

\author{
Key Words \\ Phenoloxidase - Innate immunity $\cdot$ Hemolymph • Clotting • \\ Coagulation $\cdot$ Hemostasis $\cdot$ Insects $\cdot$ Wound healing
}

\begin{abstract}
In response to microbial infection, insects mount several defense reactions including the induction of proteolytic cascades that lead to localized melanization and coagulation. Melanization requires the activation of prophenoloxidase (proPO) to its active form phenoloxidase (PO), a key enzyme that leads to the formation of melanin at wound sites and around intruding microorganisms in the hemolymph. Clotting is critical in limiting hemolymph loss and initiating wound healing following injury; it quickly acts to form a solid barrier against infection by immobilizing microorganisms and promoting their killing. Recent advances in Drosophila and other insects imply a possible link between PO and the coagulation system, although the exact molecular mechanisms controlling this interaction appear to be complex and are still not well defined. The development of hemolymph experimental techniques in Drosophila larvae together with proteomic analysis have further led to the identification of proPO as a cross-linking component that is involved in the hardening and melanization of clots. However, clot PO activity varies between insect species and life stages, depending on physiological and ecological conditions. Here we review
\end{abstract}

our current knowledge of the association between PO and coagulation and discuss the implications of the previous findings on insect innate immunity and hemostasis.

Copyright $\odot 2010$ S. Karger AG, Basel

\section{Introduction}

Insects and other invertebrates lack certain characteristics of the adaptive immune system that provides vertebrates with pathogen-specific receptors and immune memory (e.g. rearrangements of immunoglobulin genes, clonal expansion, and selection); instead, they rely on innate immune responses to defend against foreign microbes [1]. Innate immune mechanisms are controlled by signaling pathways that are remarkably well conserved [2-4]. The insect immune system consists of a sophisticated panel of humoral and cellular defense mechanisms, acting at local as well as systemic levels, which together provide efficient protection against pathogenic infections [5]. Humoral defenses include the transcriptional activation of a panoply of genes that leads to the production of peptides and effector molecules with both recognition and antimicrobial properties [6,7]. Cellular responses in insects are mediated by the activity of macrophage-like blood cells, or hemocytes, that engage in several immune functions including phagocytosis, the formation of mul-

\section{KARGER \\ Fax +4161306 1234 \\ E-Mail karger@karger.ch}

www.karger.com
(C) 2010 S. Karger AG, Basel

1662-811X/11/0031-0028\$38.00/0

Accessible online at:

www.karger.com/jin
Dr. Ioannis Eleftherianos

Department of Biological Sciences

The George Washington University

336 Lisner Hall, 2023 G Street NW, Washington, DC 20052 (USA)

Tel. +1 202994 0876, Fax +1 202994 6100, E-Mail ioannise@gwu.edu 
Fig. 1. Simplified schematic representation of the cross-talk between the proPO-activating system and the coagulation system in insects following microbial challenge. PO is currently known to be involved in cross-linking and melanizing the primary clot to produce the solid, melanized, and mature clot, which plays a major role in sealing wounds and trapping microorganisms. For more details, see text.

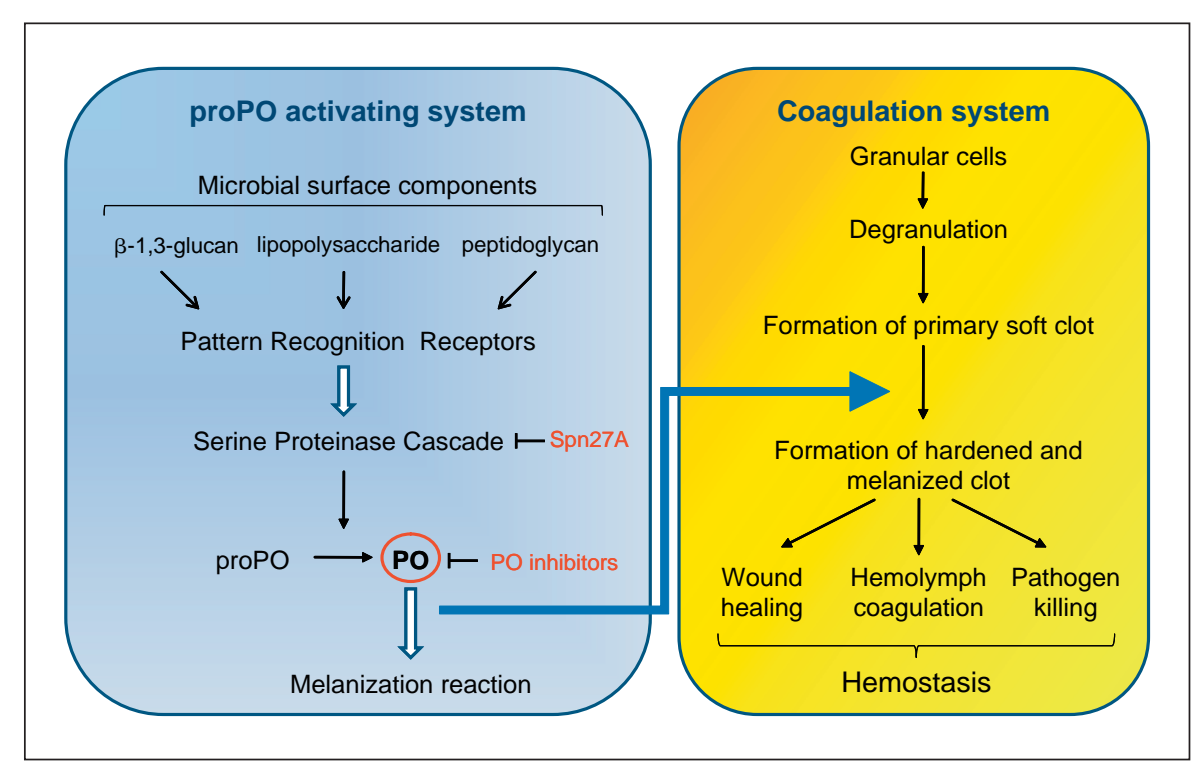

ticellular hemocyte aggregates, nodulation and encapsulation of invading bacteria and parasites, and the production of reactive intermediates of oxygen and nitrogen [8].

Proteolytic cascades play a major role in insect innate immune reactions because they can be induced more quickly than immune responses that depend on changes in gene expression [9]. Another branch of insect immunity constitutes the melanization reaction, which involves the rapid synthesis and deposition of a brownblack pigment (called melanin) at the site of infection and injury. Melanin as well as other intermediates of the cascade (e.g. quinone-like substances) possess cytotoxic activity towards microorganisms, restrain the invasion of microbial pathogens into the body cavity of the host, and assist in wound healing $[10,11]$. A key enzyme in melanin biosynthesis is phenoloxidase (PO), which catalyzes the oxidation of phenols to quinones leading to spontaneous polymerization to form insoluble melanin [12-15]. Previous studies in large insects have shown that under normal physiological conditions, $\mathrm{PO}$ exists as an inactive precursor called prophenoloxidase (proPO) and, following either physical injury or microbial challenge, it is released by specialized cells that circulate in the hemolymph (the insect blood equivalent) and is activated via limited proteolysis through the action of a serine protease cascade [16]. The melanization pathway is triggered after the recognition of microbial products through pattern recognition proteins and results in the transcription of certain genes including those that encode for serine protease inhibitors such as serpins which, through negative regula- tion, prevent premature and excessive activation (fig. 1). The significance of this immune defense in limiting infections caused by pathogenic organisms has been emphasized during the last few years $[9,17,18]$.

\section{The Coagulation Response in Insects}

The external cuticle in insects is the first line of defense that provides an effective physical barrier against the attachment and penetration of pathogens and prevents severe loss of hemolymph following injury (hemostasis) [10, 19]. Coagulation, or hemolymph clotting, is an efficient mechanism that acts to seal wounds and trap microorganisms, blocking their entry into the insect open body cavity and their subsequent spread throughout the hemocoel [20]. The invading microbes are subsequently agglutinated, immobilized, and killed by various lectins and antimicrobial peptides [19]. Coagulation is a rapid and extensive process that forms part of the innate immune system. It overlaps the humoral and cellular defenses and involves a combination of soluble and cell-derived factors [10, 21]. It mainly occurs in soft-bodied insect larvae, but not in adults with a hard cuticle, and has not been demonstrated in embryos [20, 22]. Interestingly, variation has been observed between coagulation mechanisms in different insect species, and only few similarities have been found between different life stages within species [22].

Different patterns of coagulation have been described based on the microscopic appearance of hemocytes and 
their interaction with plasma factors, which are both critical for the formation of the clot in insects [22]. In particular, granular cells and plasmatocytes are involved in the clot formation process that consists of 4 consecutive stages: (i) hemocytes (a subpopulation of granular cells) disintegrate and extracellular matrix is synthesized, which forms the primary or soft clot that is responsible for sealing the wound, (ii) the proPO pathway, together with transglutaminase, is activated and as a result the hard clot is produced, (iii) hemocytes (plasmatocytes) are set off by the plasmatocyte-spreading peptide [23, 24], turn towards the wound, and tightly cover the clot, isolating it from the hemocoel (scab development), and (iv) epidermis is regenerated and grows across the wound, eventually replacing the scab $[10,25]$. It has been suggested that although clotting and 2 cellular defenses, nodulation and encapsulation, lead to different outcomes, all 3 processes show certain similarities in the first 3 stages of the coagulation response in lepidopteran species (butterflies and moths) [10,25].

Although the molecular basis of coagulation is still unsolved, several clotting factors have recently been identified in the fruit fly Drosophila melanogaster and other insects. An unidentified hemolymph protein in the locust Locusta migratoria has previously been proposed to be one of the most abundant plasma proteins directly involved in the coagulation process [26]. It has also been shown that lipophorin particles from fourth to fifth instar larvae from Ephestia kuehniella interact with lectins and result in their assembly into coagulation products, which effectively suppress bacterial growth and prevent the spread of damaging toxins [27]. Lipophorin and other lipoproteins have consistently been found to be involved in clotting and subsequent melanization in different insect species [28-34]. In addition, lysozyme-like activity of hemolymph collected from the site of wounding was found in the American cockroach Periplaneta americana, which implies that specific immune effectors are localized near the clotting site [35]. Finally, apolipophorins were identified as candidate clotting factors in hemolymph from Drosophila larvae [36, 37]. Developing larval experimental techniques in Drosophila proved extremely useful for performing clotting studies in this genetic insect model. These methods led to the isolation of the clotting factors hemolectin and fondue [38-41], which were found by proteomic comparison [36] of hemolymph before and after coagulation using the pullout assay [37]. Interestingly, Drosophila hemolectin has a strong similarity to the von Willebrand factor that is involved in coagulation in humans [42]. Another identified coagula- tion component included the enzyme transglutaminase, which is a second mammalian clotting factor found in flies (also found in coagulation of other invertebrates) $[41,43]$.

\section{Involvement of PO in Coagulation}

Previous and recent findings strongly imply that the melanization (proPO-activating) cascade is intimately associated with factors stimulating the coagulation response in insects. PO has long been thought to play an important role in coagulation in the wax moth Galleria mellonella $[44,45]$. More recently, an in vitro coagulation reaction with cell-free hemolymph was used for isolating proteins related to hemolymph coagulation in Galleria [46]. This approach resulted in the identification of known immune players that included components of both the proPO-activating cascade and the coagulation system, supporting the idea that both responses work together during the formation of a hemolymph clot. It has further been shown that 2 major locust proteins (P260 and P85) are involved in hemolymph clotting [26]. The addition of plasma or P260-P85 complex to a hemocyte lysate supernatant before activating with a beta-1,3-glucan (laminarin) led to lower PO levels compared to the control treatment. This decrease in PO activity was not recorded when the protein complex was added to a previously activated proPO pathway. It was also reported that PO activity is linked to lipid particles and is regulated by particle-specific immune proteins in E. kuehniella. The implication of this model is that the formation of adhesive lipid particles determines the interaction of other particles (including PO) and cells, which may lead to coagulation response [27].

A previous report in Drosophila evidenced prolonged bleeding in hemolectin-silenced flies after septic injury, which was not due to defects in the PO pathway [38]. However, this study contrasts with the observation that the melanization reaction takes part in wound healing and bleeding control [47]. Another report has indirectly identified PO in the clot [36], though additional data implied that clotting occurs in the absence of $\mathrm{PO}$ and that the clot is able to bind invading bacteria [37]. Using a modified version of the pullout assay, several clotting components (including proPO) were isolated and, in combination with Drosophila mutants, the function of their respective genes was tested. Results showed that in Black cell (Bc) mutant larvae, which lack PO activity, bead aggregation was similar to or even higher than in the 
wild-type control treatment, and clot fibers were formed in hemolymph from the mutant animals. The investigation concluded that Drosophila PO may be responsible for the sclerotization/melanization of the larval coagulum and wound healing rather than for the coagulation process itself. Interestingly, a similar result was obtained for the mosquito Armigeres subalbatus [48]. However, a study examining hemolymph coagulation in aquatic larvae of the malaria vector mosquito Anopheles gambiae reported that the Anopheles larval clot is different from the Drosophila clot in terms of formation, structure and composition, and that $\mathrm{PO}$ appears to be more critical in clot formation in Anopheles than in Drosophila [33].

A subsequent report in flies revealed that $\mathrm{PO}$ is not required for initial soft clot formation, as demonstrated by its appearance in $B c$ and $l z$ mutant larvae (also lacking hemolymph PO) and that these mutants were able to stop bleeding following wounding [20]. The authors also used ex vivo methods to show that $\mathrm{PO}$ acts during clot maturation, producing a mature hard clot by cross-linking the original soft clot. In particular, PO cross-linking and melanization may control the invading bacteria, reducing the risk of infection after injury. They showed this cross-linking directly by measuring the loss of ductility in clots from wild-type flies following PO activity compared to mutant clots lacking PO, or wild-type clots formed after treatment with a PO inhibitor. However, no differences in bacterial mortality were observed between wild-type and $l z$ mutant flies, and no correlation was found between $\mathrm{PO}$ activity and bacterial killing in the clot, suggesting a possible minor role of $\mathrm{PO}$ in destroying bacterial cells. Interestingly, a separate study produced a different outcome suggesting that $\mathrm{PO}$ is required for larval wound healing in flies [49]. The difference between the outcomes of the 2 studies was attributed to possible variations in the wound's size or site, the stage of the tested larvae, or treatment after the injury [20]. To test the importance of clotting factors and PO in the Drosophila immune defense, survival assays using fly mutants have also been employed [39]. The results clearly illustrated that hemolectin mutants combined with mutations interfering with PO and the induction of antimicrobial peptides ( $B c / i m d ; ~ h m l)$ were more sensitive to infection with Gram-negative bacteria compared to double mutants ( $B c /$ imd), supporting the theory that sequestration of bacteria in the clot contributes to the prevention of infections.

Another study tested how PO is activated following injury in both Galleria and Drosophila, in which proPO is released from specialized hemocytes named oenocy- toids and crystal cells, respectively, as soon as bleeding occurs [50]. The 2 insect models elicited different melanization responses. Melanization in Galleria larvae injected with a bacterial elicitor (peptidoglycan) was consistent and the darkening of ex vivo clot preparations was similar between samples. However, melanization in Drosophila in vivo and ex vivo preparations was kept within a restricted area following treatment with peptidoglycan. It was further shown that peptidoglycan was more suitable for activating Galleria proPO while Drosophila proPO was activated at high levels using phosphatidylserine, a negatively charged phospholipid normally found on the inner surface of the plasma membrane and exposed during apoptosis. In addition, Drosophila loss-of-function mutants for the proPO inhibitor serpin 27A (Spn27A) and mutant flies expressing a dominant active form of Toll $\left(\mathrm{Tl}^{8}\right)$ that mimics immune activation were used to correlate this study with previous findings on proPO activation in flies subjected to septic injury. These mutants have been previously found to exhibit high PO activity and melanotic aggregates in the hemolymph, respectively [51, 52]. Data showed, nevertheless, that clots from both mutant strains were not melanized and, in the case of spn $27 \mathrm{~A}$ mutant flies, the lack of melanization was attributed to low levels of PO substrate in the clot. Finally, hemolymph and clot melanization patterns in spn27A mutant Drosophila and uninfected Galleria shared a high degree of similarity. These results suggest that both endogenous and foreign elicitors are able to activate the same immune response in species- and context-dependent ways [50].

The latest work on the topic [43] strongly establishes that clotting has an important immune function by limiting the dissemination of infections in Drosophila and that transglutaminase plays a major role in this process, while PO or other immune mechanisms like phagocytosis and the Toll or Imd pathways are not involved in the coagulation response (see details in Loof et al. in this issue [56]).

\section{Biological and Evolutionary Significance}

Despite the fact that insect hemolymph clotting shows certain similarities with the vertebrate blood clotting system [22], recent data agree with the theory that insect coagulation evolved independently to play a role in sealing wounds and trapping microbes before they enter into the open circulatory system, while vertebrates rely on blood clotting less extensively in order to avoid thromboses [25]. In terms of a possible link between coagulation 
and the proPO-activating systems in insects, the 2 proteolytic cascades are not obviously related as shown in other invertebrate clotting models like horseshoe crabs and crustaceans [18]. Previous findings suggest that insects might use a different mechanistic approach to assemble and activate their clotting pathways based on the recruitment of proteins with a local function (like PO) or systemic role into the coagulation system. Data from Drosophila and other insects imply that PO primarily forms a major component of the extracellular clot and, through the promotion of specific protein-protein interactions, it is capable of strengthening and stabilizing the clot, thus limiting the risk of infection after wounding (fig. 1). Interestingly, this does not necessarily apply to other insects since in Anopheles mosquitoes PO appears to be a key factor for the formation of the hemolymph clot itself [33]. This suggests that differences in ecology or life history traits between or even within species might reflect variation in the composition of hemolymph clot components. It has also been argued that PO might be more heavily involved in the clotting process rather than simply melanizing the initial soft clot in Drosophila larvae [21]. It would be of particular interest to test the evolutionary and functional connections among different components of the PO system and hemolymph clotting and/or wound healing. For example, the polypeptide spätzle has a dual role in Drosophila development and immune response [53]. Spätzle is also structurally homologous to coagulogen (the terminal substrate of the horseshoe crab hemolymph clotting system), and the C-terminal domains of both share structural homology with nerve growth factor [54], indicating a common ancestry for 2 key effector molecules in clotting, immunity and development [55]. Similar comparative studies could potentially uncover PO factors implicated in functional linkages between immunity and hemostasis.

\section{Concluding Remarks and Future Prospects}

Insects serve as excellent models for physiological, molecular, and genetic studies of coagulation as they have for other aspects of immune function. Insect coagulation has been tremendously difficult to study due to the lack of suitable assay techniques. However, the development of larval hemolymph test methods proved exceptionally useful for the detection and purification of novel clotting factors. At present, the molecular basis of coagulation in Drosophila larvae is the most thoroughly studied topic. Previous studies also suggest that the picture of how exactly $\mathrm{PO}$ is implicated in the coagulation response is complex and still incomplete. In particular, recent data indicate that clot proPO is activated proteolytically, that melanization is activated in different ways (at least in flies) at different times and places following injury, and that PO activity is used differently depending on physiological and ecological requirements. Perhaps the most consistent conclusion that can be drawn from these studies is that the primary clot is subsequently cross-linked and melanized by PO to produce the solid, mature clot (fig. 1). It would be very interesting to investigate the hemostatic mechanisms of coagulation in Drosophila adults and the effect, if any, of PO on hemolymph clotting as well as the role of microbial proteases in affecting PO activity at wound sites. Moreover, the finding that PO forms a component of the Drosophila coagulation system is both exciting and promising. Further genetic and genomic analyses in Drosophila should allow the identification and characterization of additional molecular components of the PO pathway and their potential involvement in clotting. In addition, future research on established insect models for innate immunity will further clarify the role of PO system in hemolymph clotting in the presence or absence of microbial challenge and result in a more comprehensive understanding of insect hemostasis.

\section{References}

1 Ferrandon D, Imler JL, Hetru C, Hoffmann JA: The Drosophila systemic immune response: sensing and signalling during bacterial and fungal infections. Nat Rev Immunol 2007;7:862-874.

2 Medzhitov R: Recognition of microorganisms and activation of the immune response. Nature 2007;449:819-826

3 Ishii KJ, Koyama S, Nakagawa A, Coban C, Akira S: Host innate immune receptors and beyond: making sense of microbial infections. Cell Host Microbe 2008;3:352-363.
4 Hetru C, Hoffmann JA: NF- $\kappa$ B in the immune response of Drosophila. Cold Spring Harb Perspect Biol 2010;1:a000232.

5 Lemaitre B, Hoffmann J: The host defense of Drosophila melanogaster. Annu Rev Immunol 2007;25:696-743.

6 Hetru C, Troxler L, Hoffmann JA: Drosophila melanogaster antimicrobial defense. J Infect Dis 2003; 187:S327-S334.

7 Brennan CA, Anderson KV: Drosophila: the genetics of innate immune recognition and response. Annu Rev Immunol 2004;22:457483.
8 Strand MR: The insect cellular immune response. Insect Sci 2008;15:1-14.

$\checkmark 9$ Cerenius L, Kawabata S, Lee BL, Nonaka M, Söderhäll K: Proteolytic cascades and their involvement in invertebrate immunity. Trends Biochem Sci 2010;35:578-583.

10 Jiravanichpaisal P, Lee BL, Söderhäll K: Cellmediated immunity in arthropods: hematopoiesis, coagulation, melanization and opsonization. Immunobiology 2006;211: 213-236. 
-11 Nappi AJ, Christensen BM: Melanogenesis and associated cytotoxic reactions: applications to insect innate immunity. Insect Biochem Mol Biol 2005;35:443-459.

12 Söderhäll K, Cerenius L: Role of the prophenoloxidase-activating system in invertebrates. Curr Opin Immunol 1998;10:23-28.

$\checkmark 13$ Nappi AJ, Ottaviani E: Cytotoxicity and cytotoxic molecules in invertebrates. Bioessays 2000;22:469-480.

14 Cerenius L, Söderhäll K: The prophenoloxidase-activating system in invertebrates. Immunol Rev 2004;198:116-126.

15 Christensen BM, Li J, Chen CC, Nappi AJ: Melanization immune responses in mosquito vectors. Trends Parasitol 2005;21:192-199.

- 16 Kanost MR, Jiang H, Yu XQ: Innate immune responses of a lepidopteran insect, Manduca sexta. Immunol Rev 2004;198:97-105.

$\checkmark 17$ Zou Z, Shin SW, Alvarez KS, Kokoza V, Raikhel AS: Distinct melanization pathways in the mosquito Aedes aegypti. Immunity 2010;32:41-53.

$\checkmark 18$ Cerenius L, Lee BL, Söderhäll K: The proPOsystem: pros and cons for its role in invertebrate immunity. Trends Immunol 2008;29: 263-271.

19 Muta T, Iwanaga S: The role of hemolymph coagulation in innate immunity. Curr Opin Immunol 1996;8:41-47.

-20 Bidla G, Lindgren M, Theopold U, Dushay MS: Hemolymph coagulation and phenoloxidase in Drosophila larvae. Dev Comp Im munol 2005;29:669-679.

-21 Theopold U, Li D, Fabbri M, Scherfer C, Schmidt O: The coagulation of insect hemolymph. Cell Mol Life Sci 2002;59:363-372.

22 Dushay MS: Insect hemolymph clotting. Cell Mol Life Sci 2009;66:2643-2650.

23 Lavine MD, Strand MR: Insect hemocytes and their role in immunity. Insect Biochem Mol Biol 2002;32:1295-1309.

24 Strand MR: The insect cellular immune response. Insect Sci 2008;15:1-14.

25 Theopold U, Schmidt O, Söderhäll K, Dushay MS: Coagulation in arthropods: defence, wound closure and healing. Trends Immunol 2004;25:289-294.

-26 Duvic B, Brehélin M: Two major proteins from locust plasma are involved in coagulation and are specifically precipitated by laminarin, a beta-1,3-glucan. Insect Biochem Mol Biol 1998;28:959-967.

-27 Rahman MM, Ma G, Roberts HL, Schmidt O: Cell-free immune reactions in insects. J Insect Physiol 2006;52:754-762.

28 Brehélin M: Hemolymph coagulation in Locusta migratoria: evidence for a functional equivalent of fibrinogen. Comp Biochem Physiol B 1979;62B:329-334.

29 Gellissen G: Lipophorin as the plasma coagulen in Locusta migratoria. Naturwissenschaften 1983;70:45-46.
30 Barwig B: Isolation and characterization of plasma coagulen (PC) of the cockroach Leucophaea madera (Blatteria). J Comp Physiol B 1985; 155:135-143.

31 Chino H, Hirayama Y, Kyomoto Y, Downer RGH, Takahashi K: Spontaneous aggregation of locust lipophorin during hemolymph collection. Insect Biochem 1987;17:89-97.

32 Lee KM, Lee KY, Choi HW, Cho My, Kwon TH, Kawabata S, Lee BL: Activated phenoloxidase from Tenebrio molitor larvae enhances the synthesis of melanin by using a vitellogenin-like protein in the presence of dopamine. Eur J Biochem 2000;267:36953703.

33 Agianian B, Lesch C, Loseva O, Dushay MS: Preliminary characterization of hemolymph coagulation in Anopheles gambiae larvae. Dev Comp Immunol 2007;31:879-888.

34 Altincicek B, Stotzel S, Wygrecka M, Preissner KT, Vilcinskas A: Host-derived extracellular nucleic acids enhance innate immune responses, induce coagulation, and prolong survival upon infection in insects. J Immunol 2008; 181:2705-2712.

35 Haine ER, Rolf J, Siva-Jothy MT: Functional consequences of blood clotting in insects. Dev Comp Immunol 2007;31:456-464.

36 Karlsson C, Korayem AM, Scherfer C, Dushay MS, Theopold U: Proteomic analysis of the Drosophila larval hemolymph clot. J Biol Chem 2004;279:52033-52041.

37 Scherfer C, Karlsson C, Loseva O, Bidla G, Goto A, Havemann J, Dushay MS, Theopold U: Isolation and characterization of hemolymph clotting factors in Drosophila melanogaster by a pullout method. Curr Biol 2004; 14:625-629.

- 38 Goto A, Kadowaki T, Kitagawa Y: Drosophila hemolectin gene is expressed in embryonic and larval hemocytes and its knock down causes bleeding defects. Dev Biol 2003;264: 582-591.

- 39 Lesch C, Goto A, Lindgren M, Bidla G, Dushay MS, Theopold U: A role for hemolectin in coagulation and immunity in Drosophila melanogaster. Dev Comp Immunol 2007;31:1255-1263.

40 Scherfer C, Qazi MR, Takahashi K, Ueda R, Dushay MS, Theopold U, Lemaitre B: The Toll immune-regulated Drosophila protein Fondue is involved in hemolymph clotting and puparium formation. Dev Biol 2006;295: 156-163.

41 Lindgren M, Riazi R, Lesch C, Wilhelmsson C, Theopold U, Dushay MS: Fondue and transglutaminase in the Drosophila larval clot. J Insect Physiol 2008;54:586-592.

-42 Goto A, Kumagai T, Kumagai C, Hirose J, Narita H, Mori H, Kadowaki T, Beck K, Kitagawa Y: A Drosophila haemocyte-specific protein, hemolectin, similar to human von Willebrand factor. Biochem J 2001;359:99108 .
43 Wang Z, Wilhelmsson C, Hyrsl P, Loof TG, Dobes P, Klupp M, Loseva O, Mörgelin M, Iklé J, Cripps RM, Herwald H, Theopold U: Pathogen entrapment by transglutaminase a conserved early innate immune mechanism. PLoS Pathog 2010;6:e1000763.

44 Rowley AF, Ratcliffe NA: The granular cells of Galleria mellonella during clotting and phagocytic reactions in vitro. Tissue Cell 1976;8:437-446.

45 Bohn H: Hemolymph clotting in insects; in Brehélin M (ed): Cells, Molecules, and Defense Reactions. Berlin, Springer, 1986, pp 188-207.

46 Li D, Scherfer C, Korayem AM, Zhao Z, Schmidt O, Theopold U: Insect hemolymph clotting: evidence for interaction between the coagulation system and the prophenoloxidase activating cascade. Insect Biochem Mol Biol 2002;32:919-928.

47 Rämet M, Lanot R, Zachary D, Manfruelli P: JNK signaling pathway is required for efficient wound healing in Drosophila. Dev Biol 2001;241:145-156.

48 Lai SC, Chen CC, Hou RF: Immunolocalization of prophenoloxidase in the process of wound healing in the mosquito Armigeres subalbatus (Diptera: Culicidae). J Med Entomol;39:266-274.

49 Galko MJ, Krasnow MA: Cellular and genetic analysis of wound healing in Drosophila larvae. PLoS Biol 2004:2:e239.

50 Bidla G, Hauling T, Dushay MS, Theopold U: Activation of insect phenoloxidase after injury: endogenous versus foreign elicitors. J Innate Immun 2009;1:301-308.

51 Ligoxygakis P, Pelte N, Ji C, Leclerc V, Duvic B, Belvin M, Jiang H, Hoffmann JA, Reichhart JM: A serpin mutant links Toll activation to melanization in the host defence of Drosophila. EMBO J 2002;21:6330-6337.

52 Zettervall CJ, Anderl I, Williams MJ, Palmer R, Kurucz E, Ando I, Hultmark D: A directed screen for genes involved in Drosophila blood cell activation. Proc Natl Acad Sci USA 2004;101:14192-14197.

53 Imler JL, Zheng L: Biology of Toll receptors: lessons from insects and mammals. J Leukoc Biol 2003;74:18-26.

54 Mizuguchi K, Parker JS, Blundell TL, Gay NJ: Getting knotted: a model for the structure and activation of Spätzle. Trends Biochem Sci 1998;23:239-242.

55 Krem MM, Di Cera E: Evolution of enzyme cascades from embryonic development to blood coagulation. Trends Biochem Sci 2002;27:67-74

56 Loof TG, Schmidt O, Herwald H, Theopold $\mathrm{U}$ : Coagulation systems of invertebrates and vertebrates and their roles in innate immunity: the same side of two coins? J Innate Immun 2011 DOI: 10.1159/000321641. 